NASA Technical Memorandum 83412

\title{
Dynamic Distortion in a Short S-shaped Subsonic Diffuser With Flow Separation
}

Richard Stumpf, Harvey E. Neumann, and Charles C. Giamati

Lewis Research Center

Cleveland, Ohio

Prepared for the

Nineteenth Joint Propulsion Conference cosponsored by the AIAA, SAE, and ASME Seattle, Washington, June 27-29, 1983

\section{NRSA}


DYNAMIC DISTORTION IN A SHORT S-SHAPED

SUBSONIC DIFFUSER WITH FLOW SEPARATION

by Richard Stumpf, Harvey E. Neumann, and Charles C Giamati

National Aeronautics and Space Administration

Lewis Research Center

Cleveland, Ohio 44135

Abstract

An experimental investigation of the time varying distortion at the diffuser exit of a subscale HiMAT forebody and inlet was conducted at Mach 0.9 in the Lewis 8 by 6 Foot Supersonic Wind Tunnel. A transitory separation was detected within the subsonic diffuser. Vortex generators were installed to eliminate the flow separation. This paper will present results from a study of the instantaneous pressure variations at the diffuser exit. The time unsteady total pressures at the diffuser exit are computer interpolated and presented in the form of a movie showing the transitory separation. Limited data showing the instantaneous distortion levels is also presented.

\section{Nomenclature}

B base radial factor (function of IDR)

d distance from surface

H annular height at diffuser exit station ( $t$ ip radius-hub radius)

IDC circumferential distortion index

IDR radial distortion index

$\mathrm{Kc}$ circumferential sensitivity factor

$\mathrm{Kr}$ radial sensitivity factor

$P$ total pressure

$\mathrm{R}$ radius

axial distance

$\triangle P R S$ distortion index, $\triangle P R S=K C B(I D C)+K r(I D R)$

Subscripts:

0 free stream

2 diffuser exit station

\section{Introduction}

The inlet designer for tactical aircraft is continually concerned with increasing the inlet performance, i.e., the inlet recovery, the tolerance to angle of attack, extending the operating range,etc, while maintaining acceptable compatibility with the engine. Many of the current inlet systems have acceptable inlet recovery and achieve the necessary compatibility by using an inlet design that employs relatively long and straight inlet ducts. These ducts obviously lead to fairly conservative designs. The demand for improved performance and minimal weight has led to an increased interest in relatively short inlets with large offsets, short lengths, and large curva-

tures. These inlets have applications in advanced turboprop inlets, VSTOL inlets, and for modern advanced technology tactical aircraft such as that investigated by the Highly Maneuverable Aircraft Technology (HIMAT) program.
The HIMAT remotely piloted research vehicle, RPRV, is an example where vehicle design considerations have imposed severe geometric constraints on the inlet. Although most major components of this RPRV were scaled from that of a man-sized high performance aircraft system, the inlet's geometric characteristics had to be compromised because of aircraft volume and engine considerations at the scaled-down size. The underbelly inlet is a normal shock, fixed geometry inlet. The cross section is contoured to conform to the forebody. Compared with other similar inlets, such as the F-16, this HiMAT inlet is significantly shorter, has increased diffusion, and sharper "S" wall curvatures. It has been shown in Ref. 1 that these characteristics have led to duct separation in the subsonic diffuser. The pressure recovery of the HiMAT inlet has in general remained good despite this separation.

In evaluating the performance of an inlet, both the inlet steady state total pressure recovery and the instantaneous distortion are impor tant. The instantaneous level of distortion must be compared with that predetermined level of engine distortion which is sufficent to cause engine stall or surge. The instantaneous pressure patterns or contours which the engine will encounter are also of interest to the engine manufacturer in determining the system compatibility.

This paper will present experimental results showing the effect of a transitory separation on the instantaneous pressure variations at the diffuser exit. The unsteady nature of the pressure fluctuations are shown by tracking the mean pressure contour and the region of transitory flow separation. The effect on the inlet recovery and instantaneous dynamic distortion are also presented.

\section{Apparatus and Procedure}

Inlet Details

The RPRV HIMAT was designed for operation to Mach numbers in excess of 1.5 . The test configuration was a 0.2884 scale model of the forebody and inlet. It was tested to Mach 1.4 over a range of pitch and yaw angles and a range of engine air-

flows. Figure 1 shows the test model installed in the test section of the NASA Lewis 8 by 6 Foot Supersonic Wind Tunnel. Geometric details of the inlet configuration can be obtained from Ref. 1. The inlet was coupled to a coldpipe-choked plug assembly which maintained choked flow conditions a short distance downstream of the compressor face instrumentation. The HiMAT inlet does not incorporate vortex generators, but a vortex generator study was conducted during the test. The design of the vortex generators is given in Ref. 1. The installation of the vortex generators in the inlet is shown in Fig. 2 . 


\section{Instrumentation}

The primary total pressure instrumentation is shown in the cutaway sketch in Fig. 3. The inlet surface nearest to the forebody will be refered to as the ramp surface in this report and the opposite surface will be refered to as the cowl surface. Four boundary layer rakes were placed in the diffuser on the ramp surface to detect the expected flow separation. The upstream rake was placed ahead of the anticipated separation whereas the other three rakes were used to define the separation region. The rakes were staggered circumferentially to avoid interference effects on downstream instrumentation. Forty combination steady state-dynamic total pressure probes were used at the compressor face to measure the diffuser exit conditions. These probes were arranged in a 5 ring by 8 rake configuration. The pressure probes were located at the center of equal areas. Only the fluctuating component of pressure was recorded from each dynamic probe. The fluctuating component of each pressure transducer was recorded on FM multiplexed tape. This component was later added to the corresponding time averaged (steady state) pressure during data analysis. The frequency response of the dynamic compressor face probes was flat to about $2000 \mathrm{~Hz}$. All analysis of the dynamic data to obtain distortion indexes was performed by Air Force Flight Dynamics Laboratory (AFFDL) personnel using DYNADEC as described in Ref. 2 .

\section{Data Reduction}

An engine cannot respond to extremely high frequency pressure pulses. It is therefore necessary to filter the pressure fluctuations to the maximum frequency that the engine can respond to. The maximum frequency that the full scale GE J85-21 engine will respond to is 250 hertz. Since the inlet model is a 0.2884 scale, the sub-scale frequency is nearly 1000 hertz. For the purposes of this test the pressure fluctuations were filtered using a low pass filter of 1000 hertz for the subscale model. The pressure fluctuations were sampled by the digitizer 5 times per cycle, or at a rate of 5000 samples per sec. These digitized pressure samples became the basis of the study of the instantaneous pressure variations associated with the transitory separation at the diffuser exit.

The analysis procedure for studying the dynamic pressure fluctuations is pictorally illus trated in Fig. 4. A digital image of the instantaneous pressures is generated on the color CRT screen in three steps. Since the number of data values for any one two-dimensional grid of digitized pressures is small, standard interpolation methods are used to generate a larger array of values for display. These values are then stored in a square array of digital memory which is in a one-to-one correspondence with the square array of picture elements (pixels) on the screen face. Finally display hardware continually scans the contents of the memory and translates memory values into colors, creating a t.v. raster image. The digitized pressures from each of the 40 probes were therefore assigned hues of either red, green, or blue depending on the pressure level. Red colors were assigned if the pressure was equal or less than the local mean static pressure level as determined by the wall static pressure measurements. Blue colors and its hues were assigned to pressure levels which fell between the wall static pressure level and the instantaneous spatial-mean total pressure level. Finally green colors and its hues were assigned to all pressures which were greater than the instantaneous spatial-mean total pressure level. The image processor system sequentially reads two-dimensional arrays which have been pre-stored on magnetic tape, creates a CRT image, and controls a motion camera operating in single-frame mode to photograph the CRT screen. The color representation within this paper has been replaced by cross-hatching. The frame number, the word "HIMAT", the angle of attack, and the angle of yaw are placed on each frame on the right side as shown in Fig. 4. Additional details of the image processing and the color graphic techniques can be obtained in Refs. 3 and 4 .

\section{Results and Discussion}

The scale model of the HiMAT inlet, including the forebody and canards, was tested to determine the separation characteristics and the associated effects on pressure recovery and distortion levels. The flow along the ramp surface was surveyed at four axial locations. The total pressure distributions are shown in Fig. 5 for operation at Mach 0.9 , design flow rate, and zero pitch and yaw angle. The wall static pressures obtained from Ref. 1 are included on the profiles $\left(d / R_{2}=0.0\right)$. The profile from the upstream rake is typical of a turbulent boundary layer and shows little free stream pressure loss. Each of the downstream rake profiles indicate flow separation and an attendant loss in pressure recovery. The initiation of the separation is therefore between an axial location of $x / R_{2}=9.503$ and 10.939 .

The steady state total pressure profiles at the compressor face are shown in Fig. 6 for the same conditions as the previous figure. The recovery is plotted for each of the four quadrants. The local wall static pressure ratios are also shown on the outer or tip surface, $(d / H=1.0)$. These data were measured with the mid diffuser rakes removed to eliminate any possible rake wake effects. Recovery on the cowl surface was generally very good except in the near wall region. The recovery profiles on the ramp surface clearly show a large reduction in pressure on the top centerline of the diffuser which was downstream of the flow separation. However, the near wall total pressures are higher than the measured wall static pressures indicating that flow reattachment has occured. Examination of the dynamic pressure however indicates that transitory separation is present. A typical time history of the dynamic component of total pressure obtained from a dynamic pressure probe at the compressor $f$ ace and downstream of the diffuser separation is shown in Fig. 7. The pressure trace shows a onesided character. When the mean total pressure is assigned the steady state level of pressure as independently measured, the downward spiking extends from this mean steady state total pressure level down to the steady state static pressure level. This strongly suggests that at those instants of time when downward spiking occurs in the pressure traces, a transitory separation is being detected at the compressor face. Additional creditability to this argument is obtained by noting that the upper level of the envelope of time varying pressures corresponds approximately to the mean free stream total pressure. The downward 
spiking in the pressure signals occured randomly and was not associated with any particular frequency. The severe spiking was usually noted on the probes of the outer ring and on the probes immediately downstream of the diffuser ramp separation. Occasionally, however, the spikes were noted on each of the other dynamic pressure probes. The downward spikes on these other dynamic probes did not generally extend to the static pressure levels.

The transitory separation just described was found at all operating conditions tested. The steady state performance as measured by the average recovery was however not severely impaired. The inlet was tested over a range of pitch and yaw angles. The pitch was varied from -10 degrees to +25 degrees and the yaw ranged from 0 to 10 degrees. The steady state recovery is presented in Fig. 8a for operation at Mach 0.9 and design mass flow. The contours are interpolations of the actual measured data. The recovery is good at all operating conditions. The results of the AFFDL instantaneous dynamic distortion screening are shown in Fig. $8 \mathrm{~b}$ for the operating range of pitch and yaw tested. The distortion is given in terms of the $\triangle P R S$ index. The definition of the index is given in the symbol section. Again the distortion contours shown are interpolations of the actual data. The maximum instantaneous distortion at zero yaw angle was found to occur at about 7 degrees angle of attack. The distortion increased as the inlet yaw increased. It should be noted that the peak values shown in this figure all occured during that instant of time when transitory separation was present. That is, the peak distortion occured during that instant of time when downward spiking was present on one or more dynamic pressure probes.

Vortex generators are often used to eliminate separations in ducts and inlets. They were therefore installed on both the cowl and ramp surfaces of the inlet. Details of the generator design are given in Ref. 1. A comparison of the total pressure profile at the mid-diffuser with and without vortex generators is given in Fig. 9. The conditions shown are for zero angle-of-attack and yaw, a freestream Mach number of 0.9 , and design mass flow. The profile shown for no vortex generators was presented in Fig. 4 earlier and is repeated here in Fig. 9. The profile with generators clearly indicates that the generators were successful in eliminating the mid-diffuser separation. Since the vortex generators are basicly loss devices, the installation of vortex generators caused the recovery to be slightly reduced. Significant reductions in both the circumferential and radial components of steady state distortion were noted with the use of vortex generators. The peak instantaneous distortion was not however reduced. The distortion sensitivity factors for the engine used in the HiMAT RPRV are such that the engine can tolerate higher levels of circumferential distortion when the radial distortion is large than when the the radial distortion is small. Hence, although the vortex generators reduced both components of distortion, the overall level of $\triangle$ PRS was not favorably reduced. Other engines do not have this characteristic and therefore favorable reductions could be expected in those cases.

Numerous attempts have been made to predict the instantaneous dynamic distortion. The analytic technique of Melick, 5 has been studied extensively because it offers the advantage of, at least theoretically, requiring only a minimum of one dynamic pressure probe to measure the rms level of turbulence. This is significant since the amounts of dynamic data which must be recorded for a complete experimental compatibility test is gargantuan and usually leads to extremely expensive data reduction procedures. Unlike most other methods, this method does not require recording unsteady dynamic data, formulating the instantaneous distortion parameter time history, and then processing short segments during the screening process. The Melick method only requires knowledge of some steady state parameters and the measurement of the rms level of the fluctuating component of total pressure at the compressor face. The Melick method can therefore also be conviently used for on-line screening during testing. The Melick method of Ref. 5, which is based on statistics, has been widely used on inlets that do not have a separation of the type noted in the HiMAT inlet. The method has also been the subject of an extensive study, ${ }^{6}$ to minimize the necessary instrumentation and to provide guidance for the placement of probes.

When the Melick method is used as an on-line prediction technique, it is desirable to minimize the number of probes and maintain a fixed probe configuration. Previous to the work of Ref. 6 , there has been little or no guidance as to where to place dynamic pressure probes at the compressor face to achieve good predictions with the Melick method. Generally a full complement of 40 or more probes is used in the method. It was therefore of interest to evaluate the Melick method as a screening technique during testing since the method had little or no usage with HiMAT type of separated inlets. In considering where to locate the dynamic pressure probes during testing, it was decided to take the most naive approach possible. It was assumed that nothing was known about the distortion patterns present and that the engineer had no prior expectations, so therefore a uniform distribution of probe locations was the most logi-

* cal. It was futher assumed that the number of probes used in the prediction should be limited to 12. Since the inlet had provision for 8 rakes with 5 probes on each rake, two probes were used on the top, bottom, and two side rakes. On the remaining four diagonally located rakes, one probe from each rake were used in the analysis. The rakes at the top, bottom, and sides used the two measurements obtained at the second position in from each wall. The rakes with a single measurement position used the dynamic pressure measurement obtained at the center position. This distribution of measurements then formed an axisymmetric pattern.

The rms values of the fluctuating component of total pressure were recorded and the Melick method was used to provide a prediction of the peak instantaneous distortion for a number of inlet operating conditions. The results of the prediction have been compared to the results of the DYNADEC screening analysis of the complete experimental measurements using 40 probes presented earlier. The results are presented in Fig. 10. Results are presented for configurations with and without vortex generators. The results show that the predicted results for the configuration without vortex generators are in poor agreement. This is primarily because the dynamic pressure fluctuations used in the DYNADEC analysis are not adequately described by the rms value used in the 
Melick analysis. The peak instantaneous distortion occurs during those instants of time when transitory separation is present. Since the pressure fluctuations are present only a very short time, the rms measurements of pressure reflect, for the most part, the dynamics during the nonseparated mode of operation. The dynamics of the flow associated with the transitory separated mode of operation need to be isolated from the remainder of the dynamics. A method must be devised which will adequately measure the turbulent fluctuations associated with the separation and incorporate the measurement into the statistics of the method. When the separation is eliminated by the use of vortex generators the prediction method of Melick correlates well with the experimental measurements (DYNADEC). The prediction is high

but this is due to inadequate probe placement as is shown in Ref. 4 .

The pressure pattern or contour that the engine must tolerate is necessary to completely evaluate the compatibility of an engine-inlet system. The instantaneous total pressure fluctuations at the compressor face were therefore studied by analysis of the recorded dyanmic data using the technique previously described. Four data sequences were made into movies. The sequences are sumarized in Fig. 11. The first condition at zero pitch and yaw represents the basic design operating condition. The second operating condition studied was the nominal flight operating condition for the HIMAT RPRV. The third condition is the same condition except with vortex generators. This condition is characterized by having the transitory separation eliminated by the addition of vortex generators to the inlet. The last condition is one of relatively high distortion.

A typical sequence of instantaneous pressure contours taken from the movie at a pitch of $7^{\circ}$ and no yaw is shown in Fig. 12. The operating condition represents the normal mode of operation of the HiMAT RPRV. The time interval of the sequence shown is given by the numerals in the upper right corner. The interval shown in the figure covers 43 segments in the analysis. This represents 0.0086 seconds of actual time. The sequence clearly shows the random character of the transitory separation. The transitory separation region where the pressure is less than the local static pressure, as indicated by red, moves about and is not at all steady. It is however always located approximately downstream of the steady state separation which was observed in the mid-diffuser. In the calculation of the distortion index for the conditions where transitory separation was observed at the compressor face, the peak distortion always occured during those instants of time when there was a transitory separation present. This emphasizes the importance of the dynamics for these type of subsonic diffusers. When the vortex generators were added to the diffuser, the separation was eliminated from the mid-diffuser and hence the separated regions were also eliminated from the compressor face.

Many prediction techniques assume that the pressure pattern that is associated with the peak instantaneous distortion is simply an agumentation of the steady state pattern of pressure. This assumption is usually acceptable if the distortion is primarily circumferential in nature and if the turbulence level is low. When the flow dynamics are due in large part to a transitory separation, the instantaneous pressure pattern at peak distortion can be significantly different than the steady state pattern because of the separation region. A comparison of pressure contours at the peak distortion instants of time is shown in Fig. 13. Three patterns are shown for each of the data sequences analyized. For each data sequence of Fig. 11, a contour for steady state operation, a contour associated with the peak level of instantaneous distortion, and a contour obtained from the Melick method are shown in the figure. If one ignors the important regions of transitory separation, a qualitative agreement between the mean steady state pressure contour and the mean contour of pressure at peak distortion exist in the figure for each operating condition. On the same basis, there is also a qualitative agreement between the prediction of Melick and the instantaneous patterns at peak distortion. The reason for this is quite obvious. The dynamics of the flow in the HiMAT inlet are not particularly high if one ignores the transitory separation. As a result, the Melick method which does not comprehend the dynamics associated with the transitory separation provides reasonably good correlations when the separation is not present.

\section{Summary}

An experimental investigation of the dynamic distortion at the diffuser exit of a subscale HiMAT forebody and inlet was conducted at Mach 0.9 in the Lewis 8 by 6 Foot Supersonic Wind Tunnel. The results of the study are:

1. A separation was observed in the mid diffuser. This separation caused a transitory separation to be present at the diffuser exit. The transitory separation was unsteady and moved about spatially.

2. The recovery was high at all operating conditions. The recovery was above 0.97 for most of the operating conditions at Mach 0.9 .

3. The addition of vortex generators to the inlet eliminated the mid-diffuser separation and hence also the transitory separation at the diffuser exit. The resulting circumferential and radial distortion levels were substantally reduced. The overall $\triangle P R S$ was not favorably reduced since the distortion sensitivity factor for radial distortion favors high levels of radial distortion.

4. The peak instantaneous distortion always occured at instants of time when the transitory separation was present. Time averaged measurements of the pressure dynamics, such as rms, do not accurately comprehend the turbulence present during the transitory separation. Prediction methods which are dependent on rms measurements are therefore inaccurate when separation is present.

5. The instantaneous pressure fluctuations at the diffuser exit were digitized and made into a movie showing how the pressure contours changed with time. The sequences showed the separation at the diffuser exit to move about in a very random manner. The transitory separation was generally confined to being downstream of the steady state separation which was present within the diffuser.

6. The Melick prediction for peak dynamic distortion was used during the testing as an online screening method. The method was implemented with a uniform probe spacing. The distortion levels predicted were in poor agreement with the 
experimental results obtained from a DYNADEC screening when the transitory separation was present. When vortex generators were used to eliminate the separation the Melick prediction correlated well with the experimental distortion results, although in general they overpredicted. It is believed that the overprediction is due to an inadequate probe configuration.

\section{References}

1. Neumann, H. E., Povinelli, L. A., and

Coltrin, R. E., "An Analytical and Experimental Study of a Short S-Shaped Subsonic Diffuser of a Supersonic Inlet", AIAA Paper 80-0386, Jan. 1980.

2. Marious, J. J., and Sedlock, D., "Dynamic Data Editing and Computing System (DYNADEC)", Proceedings of the AFSC Science and Engineering Symposium, Kirtland AFB, New Mexico, Oct. 1973.

3. Giamati, C., "Application of Image Processing Techniques to Fluid Flow Data Analysis", NASA TM-82760, 1981.

4. Anderson, B. H., Putt, C. W., and Giamati, C. C., "Application of Computer Generated Color Graphic Techniques to the Processing and Display of Three Dimensional Fluid Dynamic Data", NASA TM-82658, 1981.

5. Melick, H. C., Ybarra, A. H., and Bencze, D. P., "Estimating Maximum Instantaneous Distortion from Inlet Total Pressure RMS and PSD Measurements", NASA TMX-73145, 1976.

6. Schweikhard, W. G., Gupta, Y. Sen, and Schweikhard, K. A., "Prediction of Dynamic Distortion Using A Minimum of Dynamic Pressure Measurements", AIAA Paper 83-1413, June 1983. 


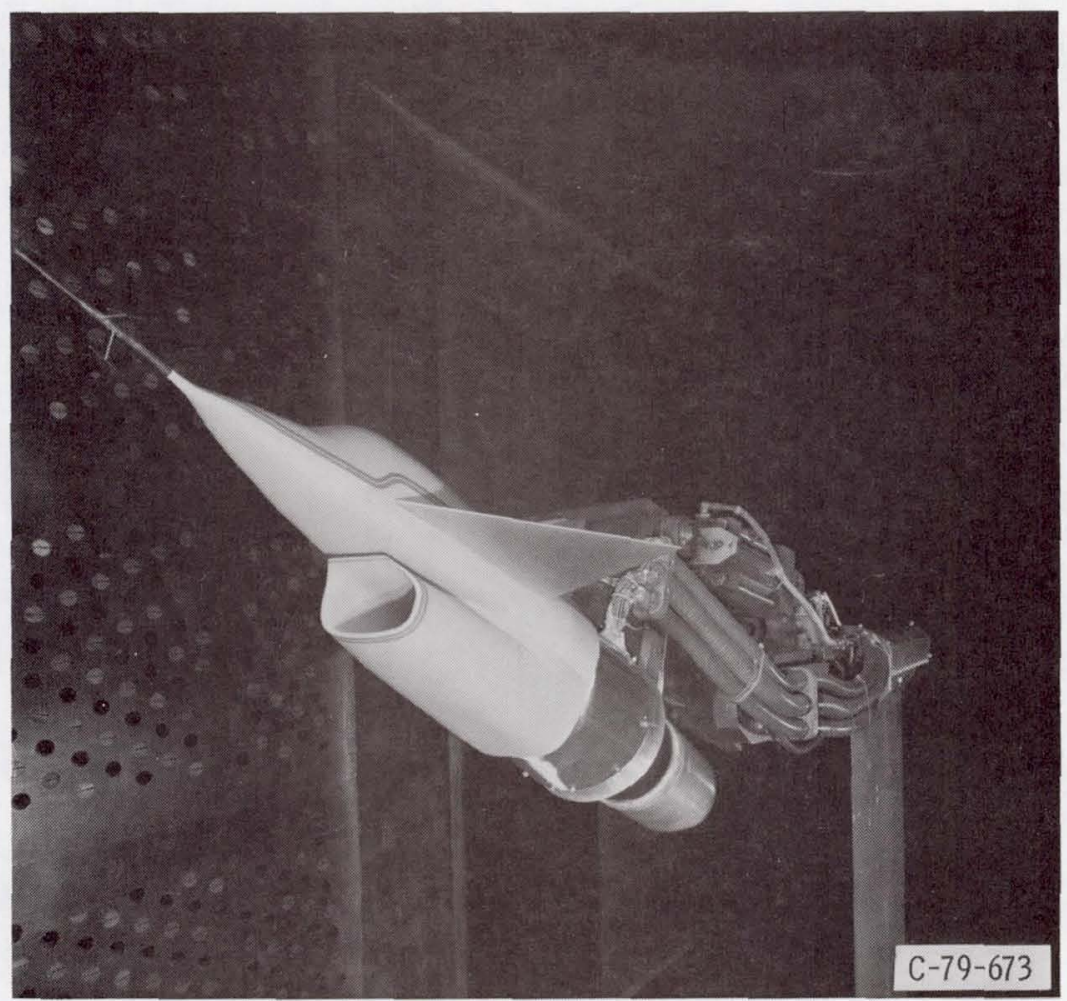

Figure 1. - HiMAT model installed in NASA LeRC $8 \times 6$ Foot Supersonic Wind Tunnel.

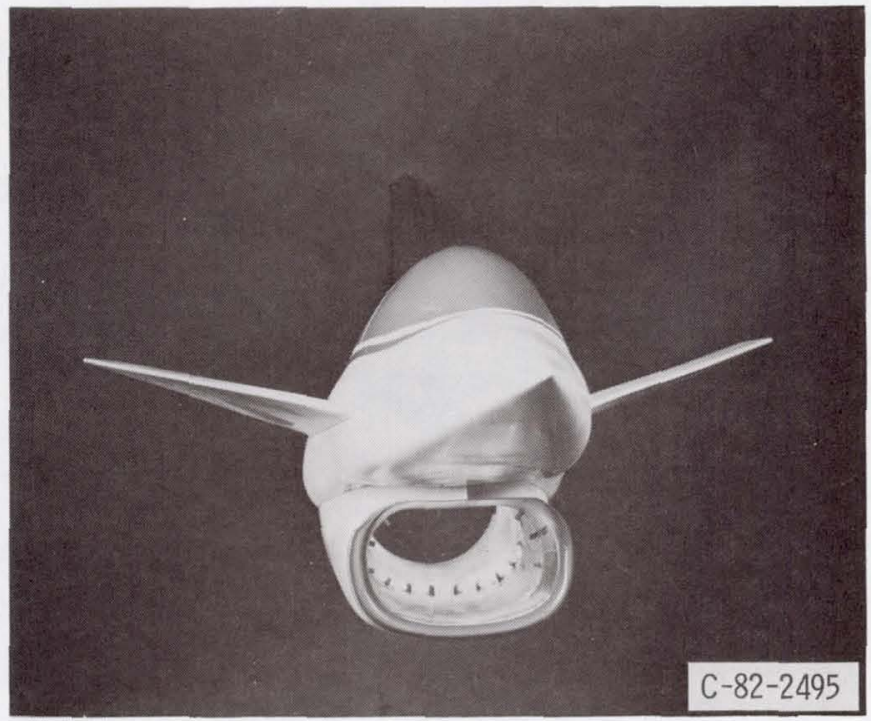

Figure 2. - Installation of the vortex generators in the HiMAT inlet. 


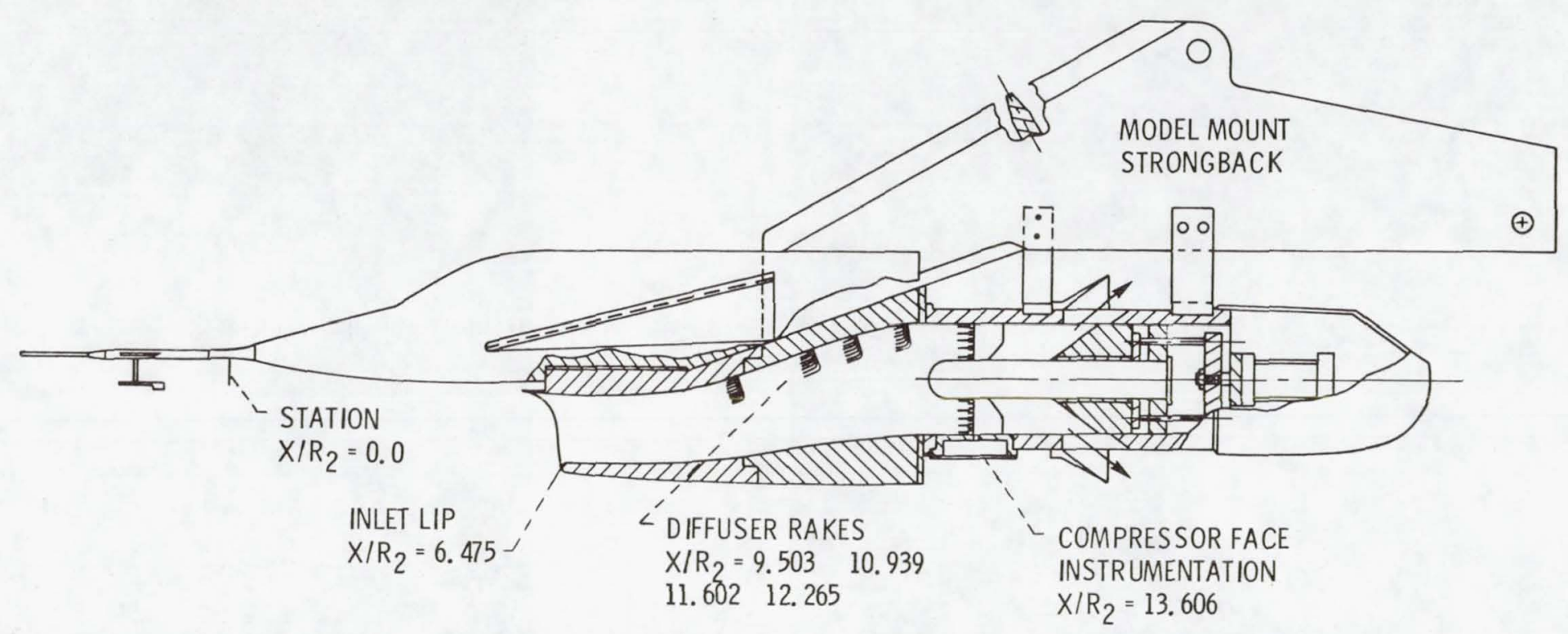

Figure 3. - Cutway illustrating inlet instrumentation and mass flow plug installation. 
DIFFUSER EXIT PRESSURE

INSTRUMENTATION

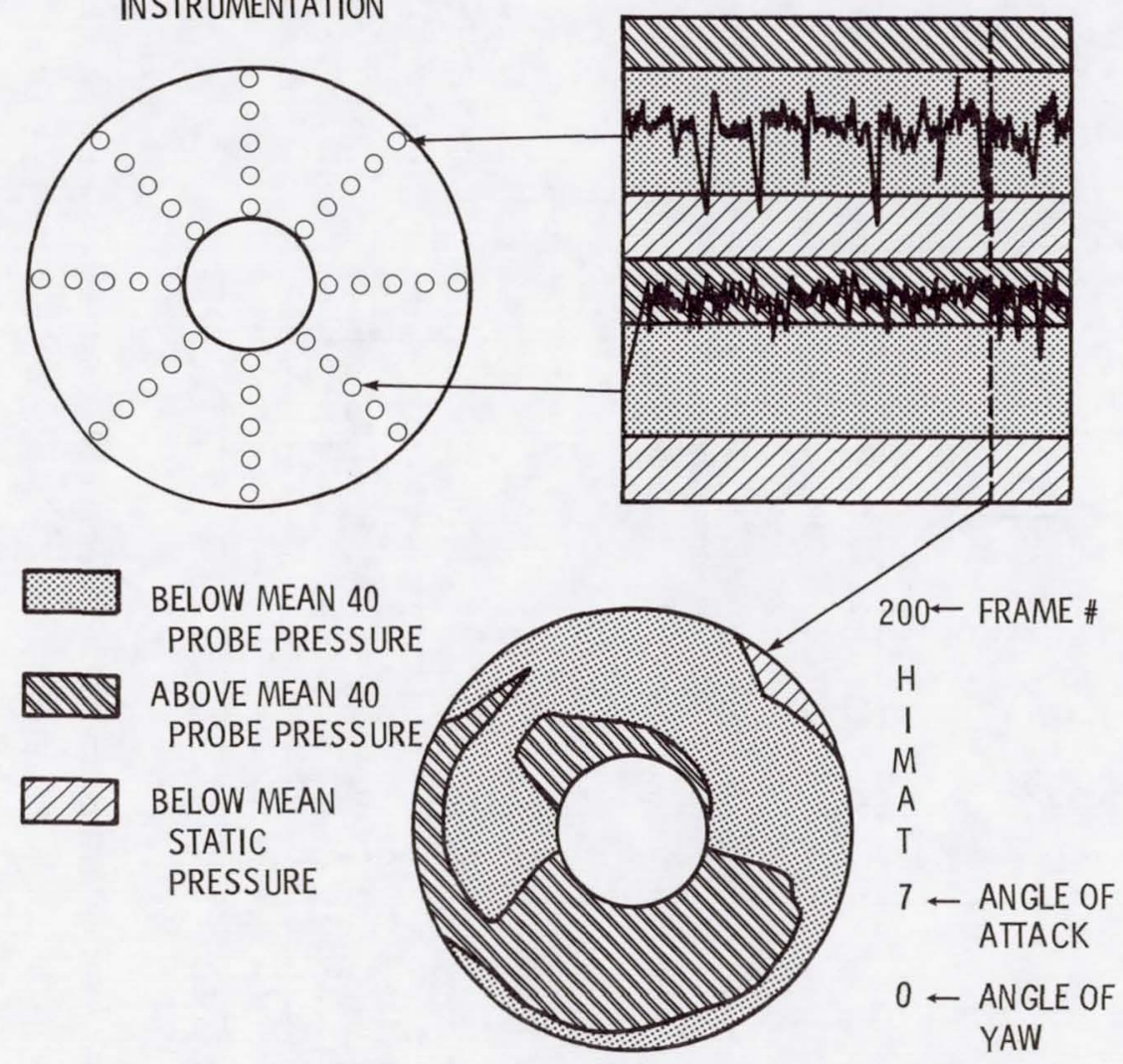

Figure 4. - Pressure contour formulation at the HIMAT diffuser exit based on experimental data.

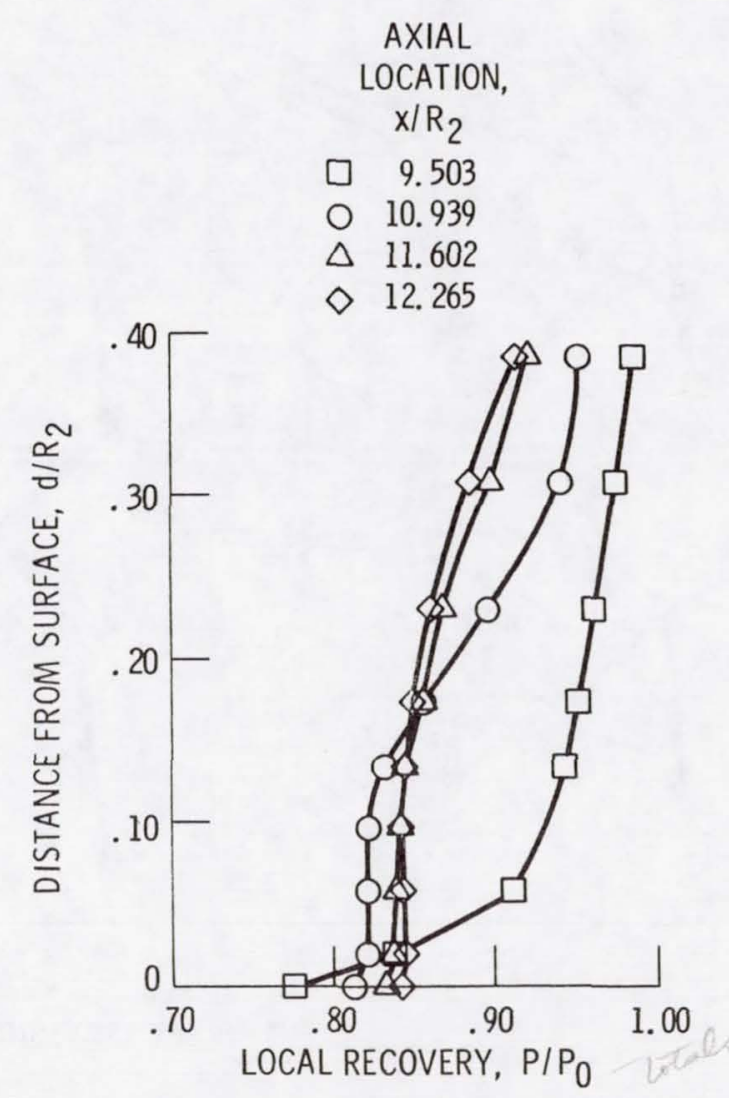

Figure 5. - Subsonic diffuser total pressure profiles; Mach number $=$ 0.9 , Corrected airflow (full scale) $=$ $56.6 \mathrm{\#} / \mathrm{sec}$, Angle-of-attack = 0 , Yaw angle $=0$. 


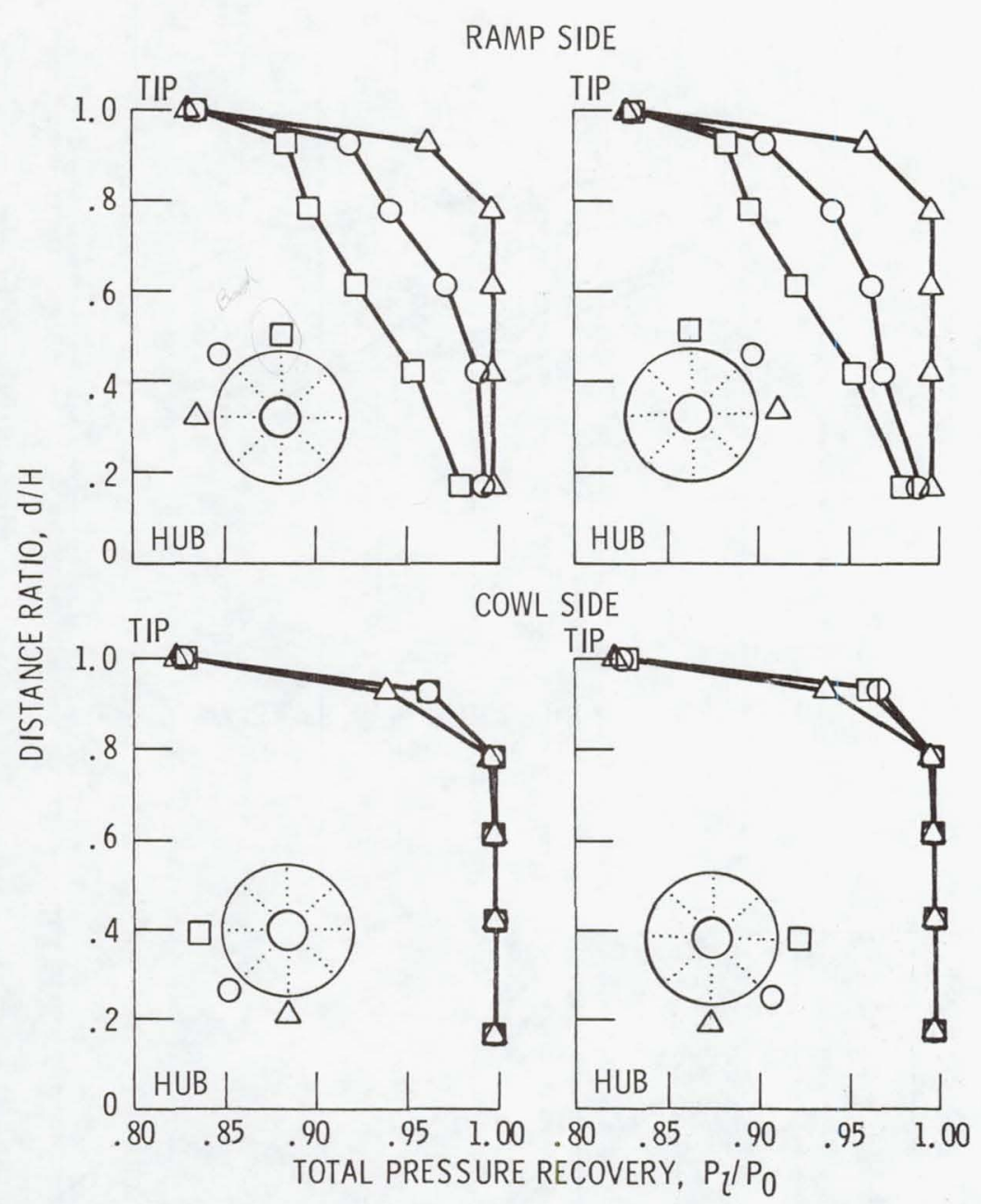

Figure 6. - Local diffuser exit total pressure recovery; Mach number $=0.9$, Corrected airflow (full scale) $=56.6 \# / \mathrm{sec}$, Angle-of-attack $=0$, Yaw angle $=0$.

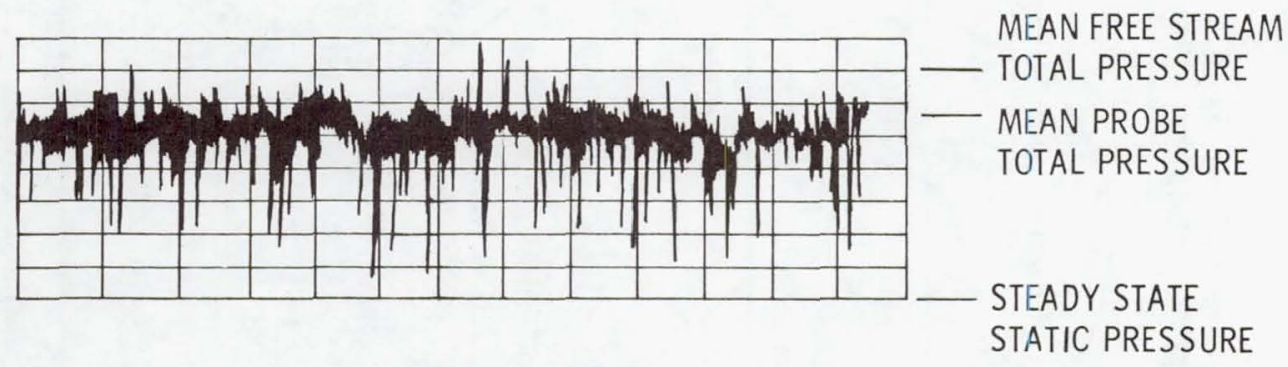

Figure 7. - Instantaneous time trace of typical total pressure variation at the compressor face. 


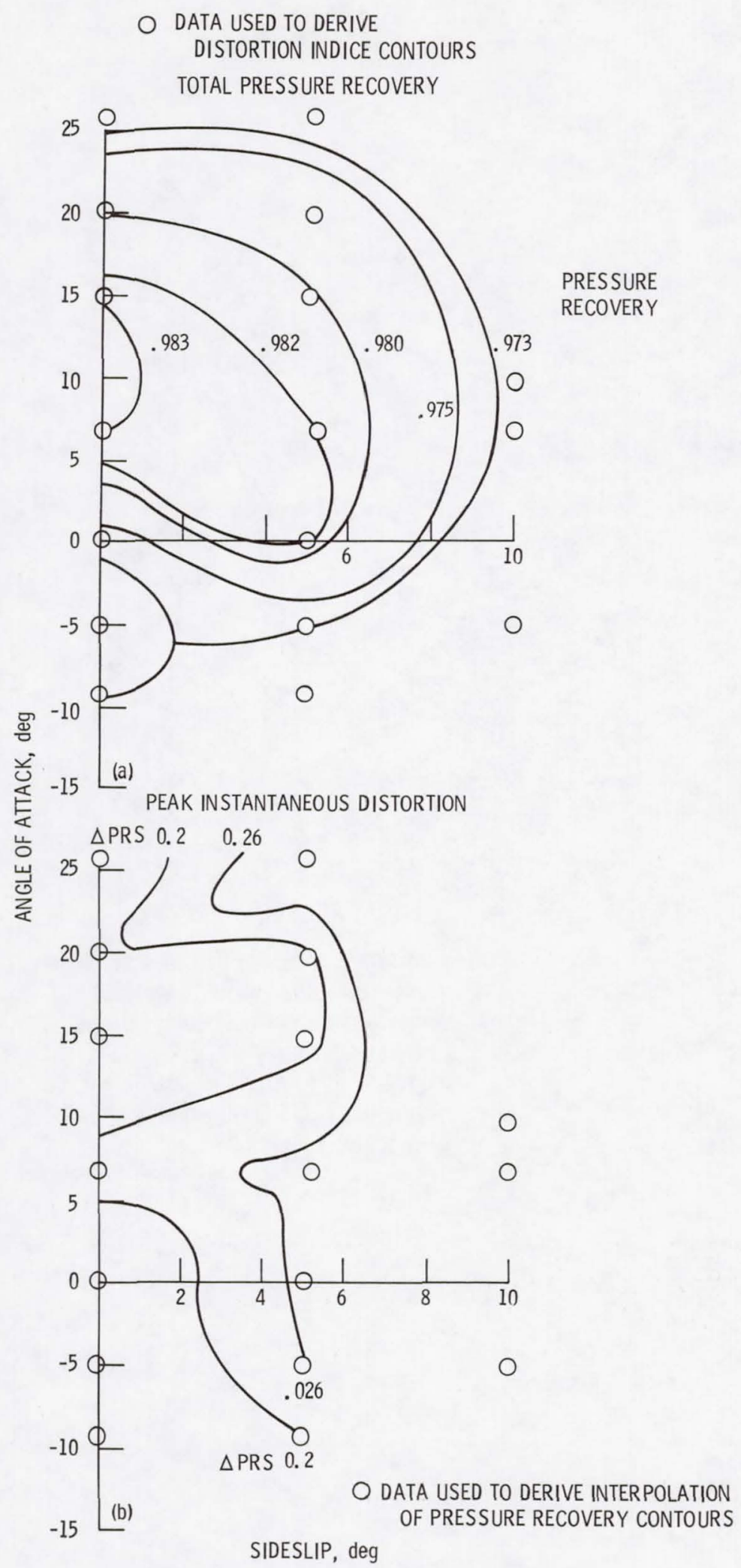

Figure 8. - HiMAT performance at pitch and yaw for operation at Mach 0.9 and inlet corrected airflow of $56.6 \# / \mathrm{sec}$. 


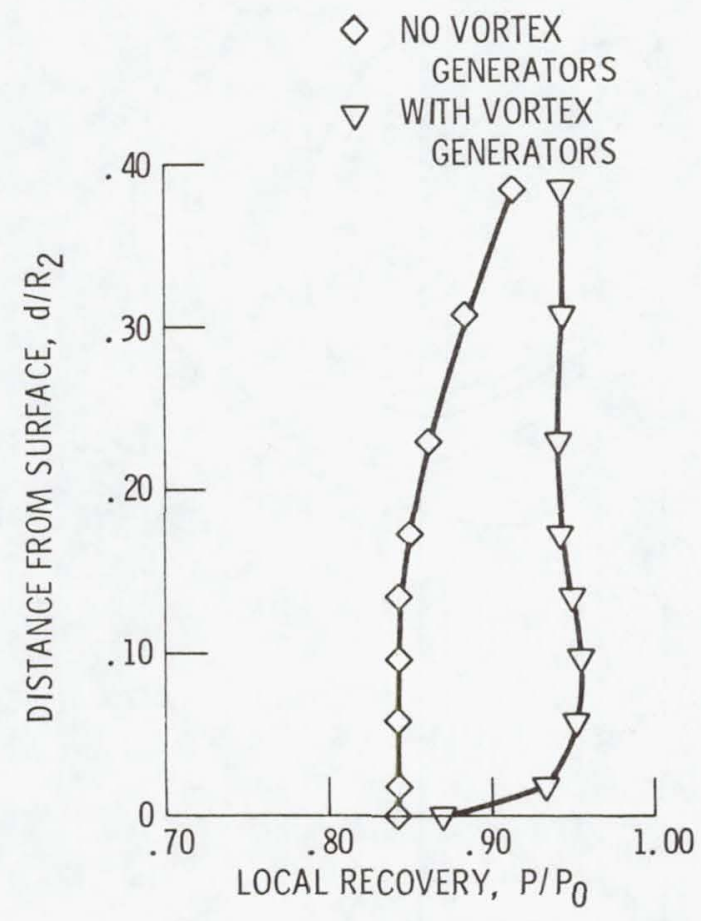

Figure 9. - Subsonic diffuser total pressure profile with and without vortex generators installed; Diffuser station $x / R_{2}=12.265$, Mach number $=0.9$, Angle-of-attack $=0$, Yaw angle $=0$, Corrected airflow (full scale) $=56.6 \mathrm{\#} / \mathrm{sec}$. 

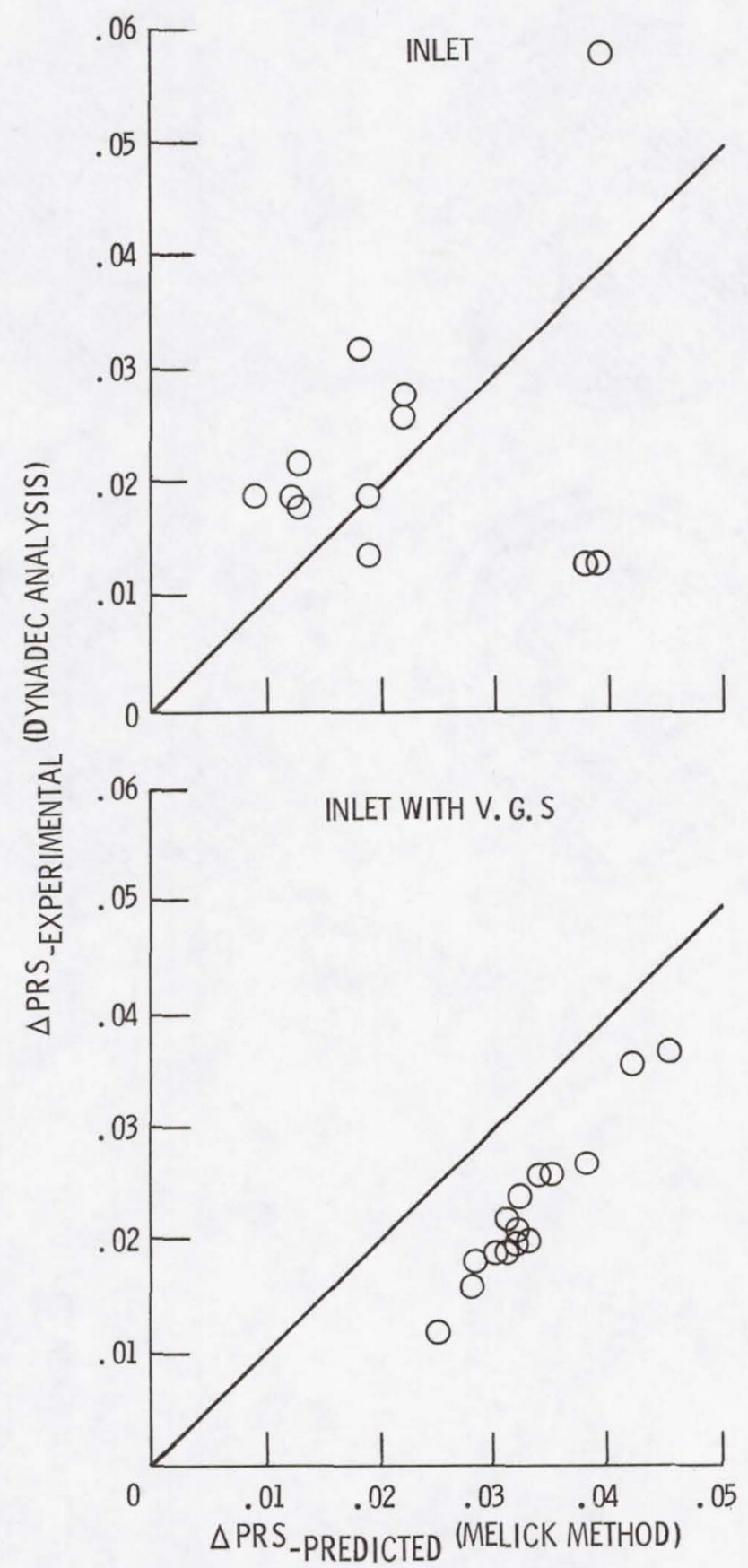

Figure 10. - Comparison of experimental and predicted peak instantaneous dynamic distortion in the HiMAT inlet at Mach 0.9. 
BELOW MEAN 40 PROBE PRESSURE

ABOVE MEAN 40 PROBE PRESSURE

Q7A BELOW MEAN STATIC PRESSURE

DATA SEQUENCE

1. ANGLE OF ATTACK 0 deg ANGLE OF YAW $\quad 0$ deg

2. ANGLE OF ATTACK $7 \mathrm{deg}$ ANGLE OF YAW $\quad 0 \mathrm{deg}$

3. ANGLE OF ATTACK $7 \mathrm{deg}$ ANGLE OF YAW 0 deg WITH VORTEX GENERATORS)

4. ANGLE OF ATTACK $7 \mathrm{deg}$ ANGLE OF YAW $\quad-5 \mathrm{deg}$

Figure 11. - Data sequences in the instantaneous pressure study at the HiMAT diffuser exit.
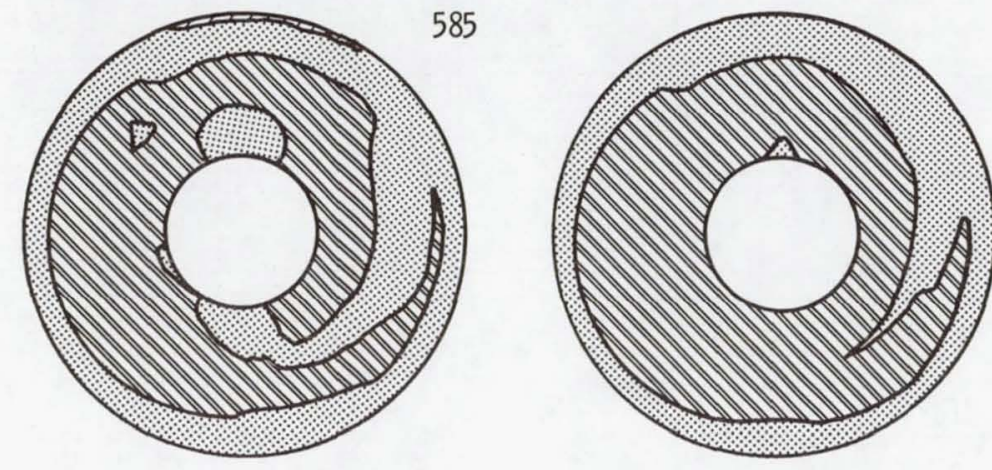

591
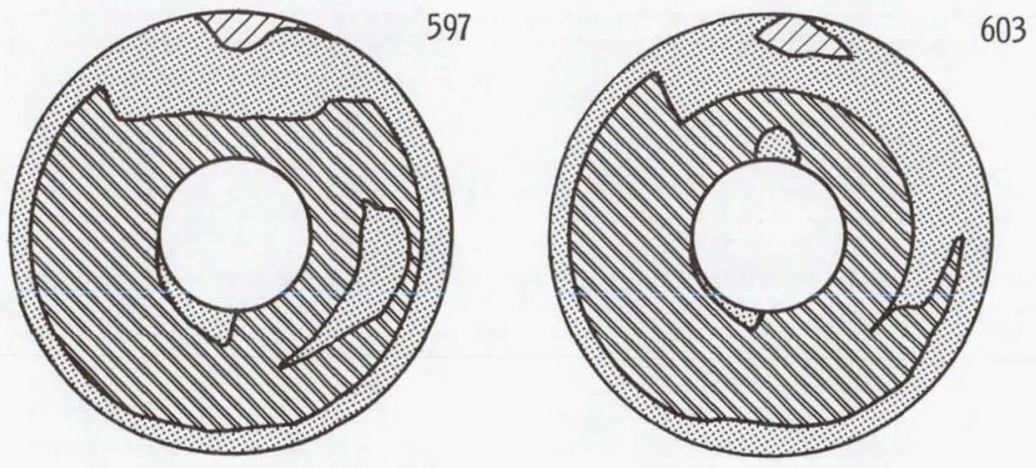

Figure 12. - Selected instantaneous total pressure contours at the diffuser exit for 7 deg pitch zero yaw with operation at Mach 0.9, and corrected airflow of $56.6 \mathrm{\#} / \mathrm{sec}$. Time increment between contours - 1.2 millisec. 
BELOW MEAN 40 PROBE PRESSURE

ABOVE MEAN 40 PROBE PRESSURE

ZIA BELOW MEAN STATIC PRESSURE
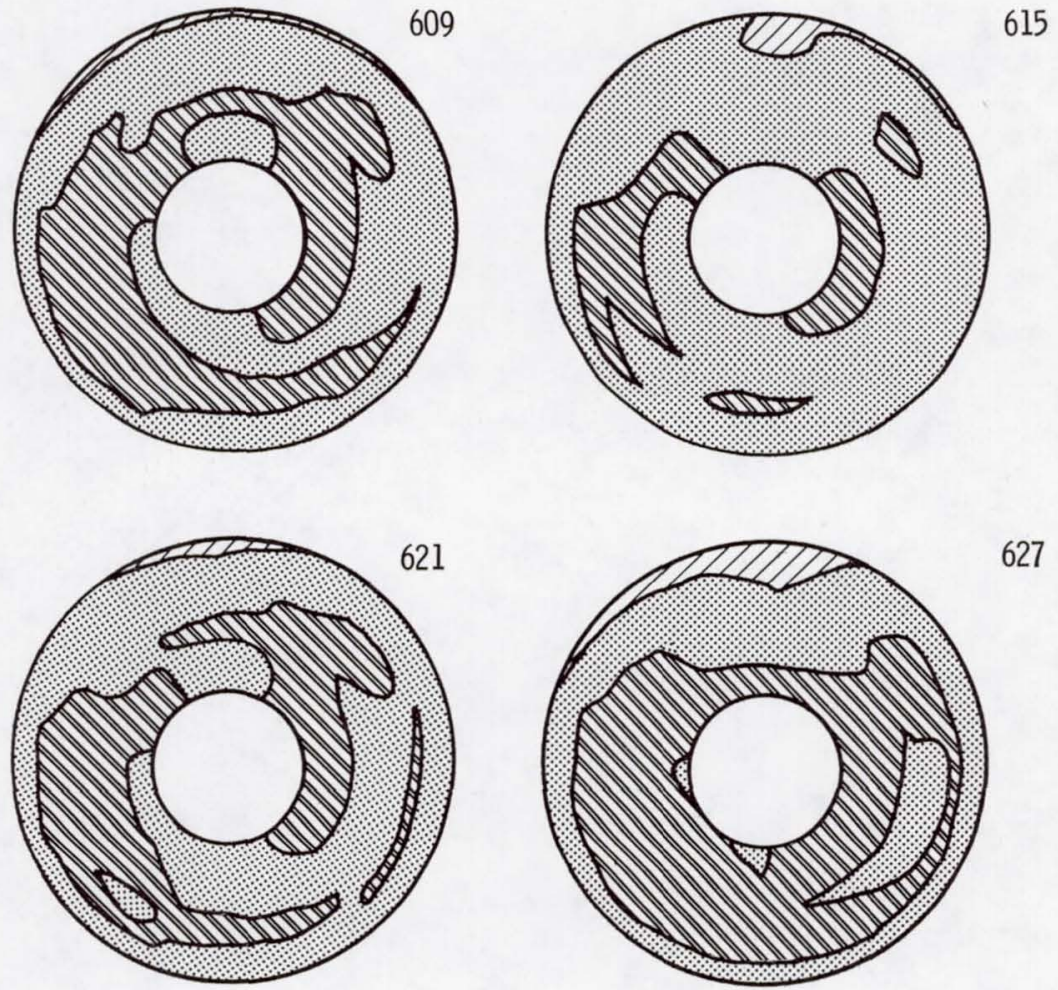

Figure 12. Concluded. 


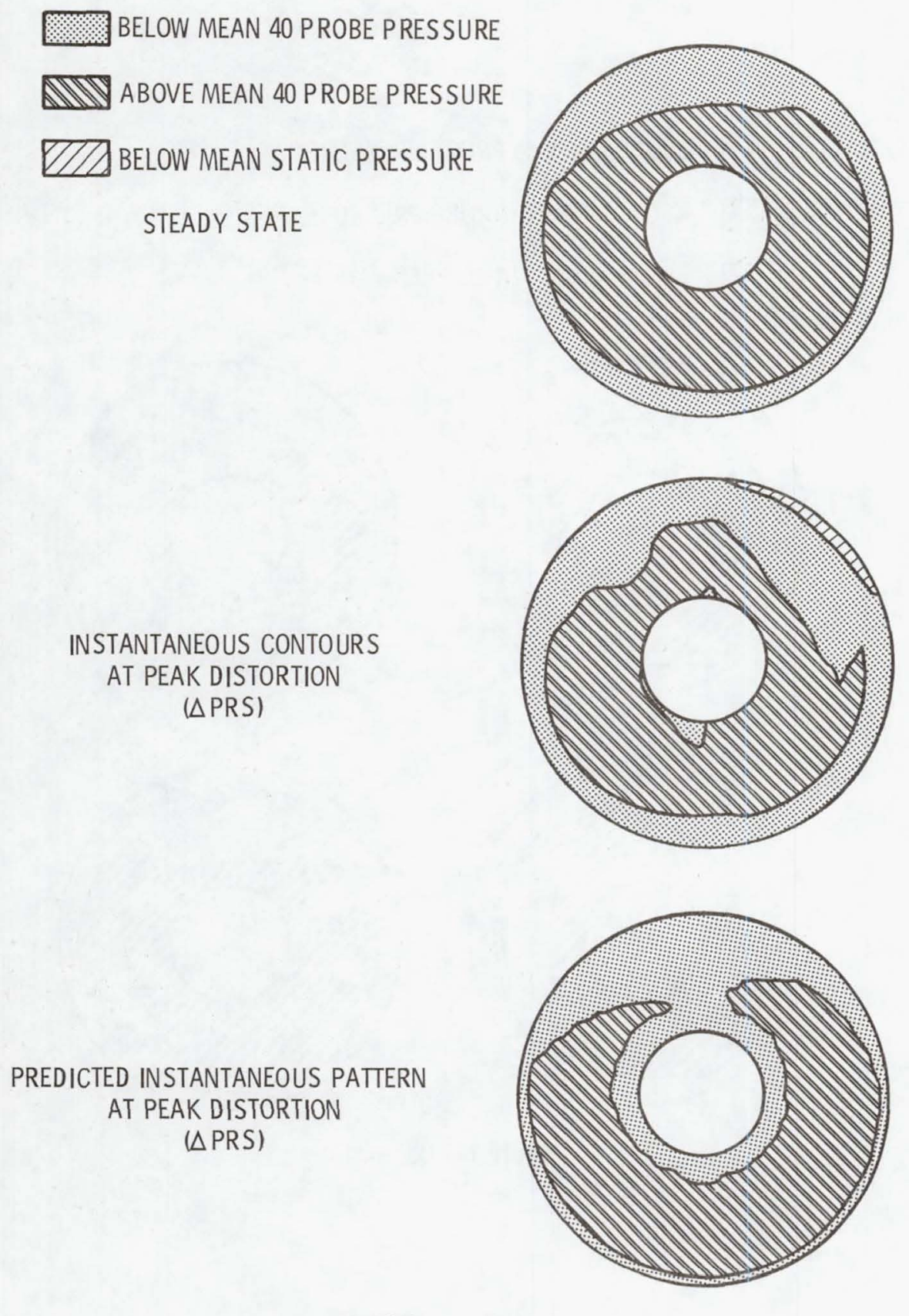

(a) Pitch $=0$ Yaw $=0$.

Figure 13. - Experimental and predicted pressure contour comparison at the HiMAT diffuser exit. 
STEADY STATE

\section{INSTANTANEOUS CONTOURS AT PEAK DISTORTION ( $\triangle P R S)$}
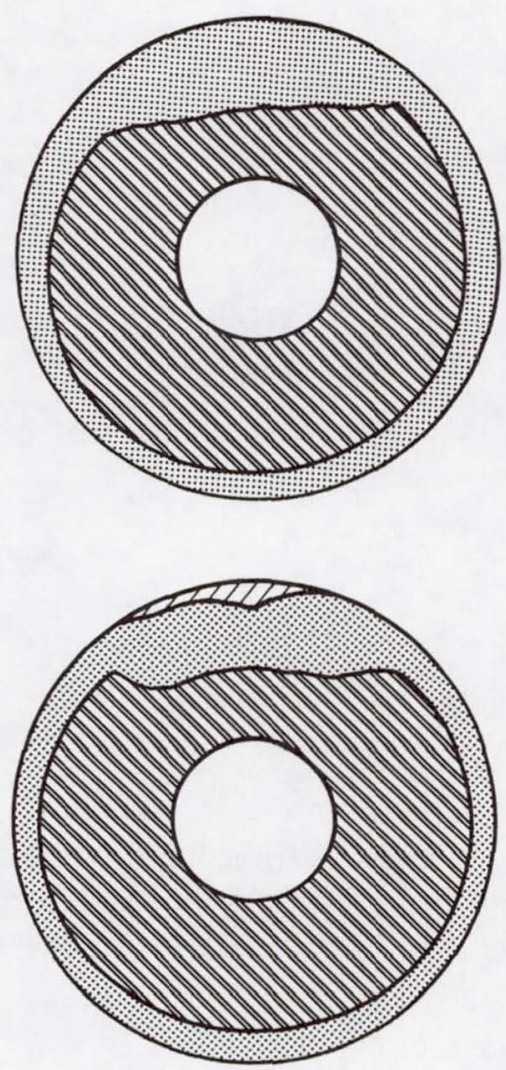

PREDICTED INSTANTANEOUS PATTERN AT PEAK DISTORTION ( $\triangle \mathrm{PRS})$

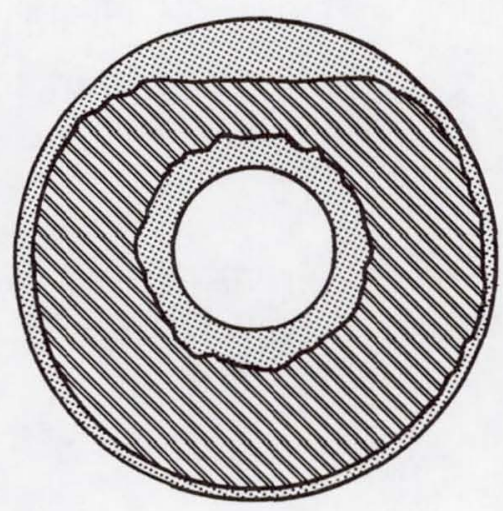

(b) Pitch $=7$ Yaw $=0$.

Figure 13. - Continued. 
STEADY STATE

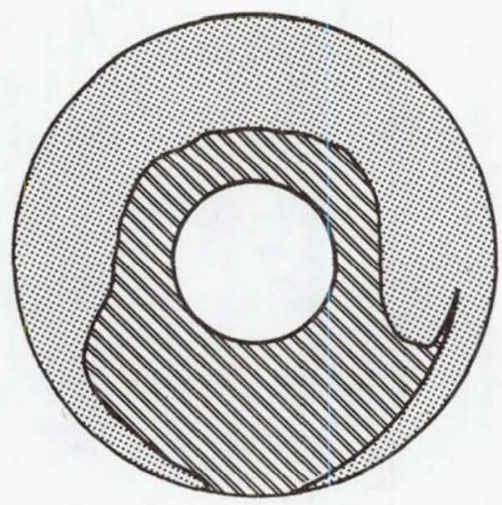

INSTANTANEOUS CONTOURS

AT PEAK DISTORTION $(\triangle P R S)$

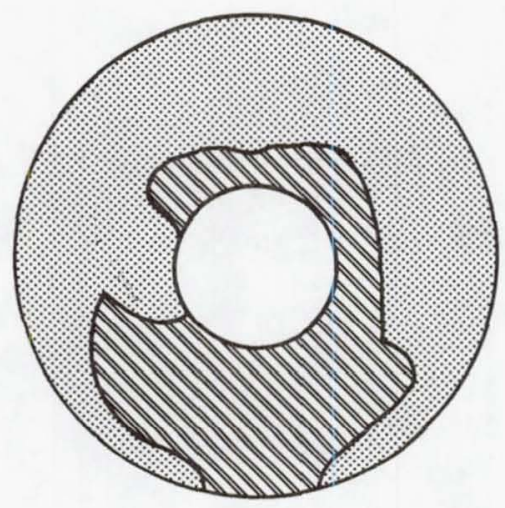

PREDICTED INSTANTANEOUS PATTERN AT PEAK DISTORTION ( $\triangle P R S)$

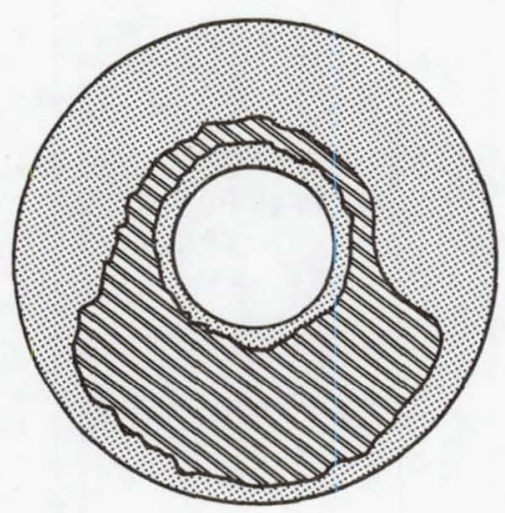

(c) Pitch $=7$ Yaw $=0$; with vortex generators.

Figure 13. - Continued. 


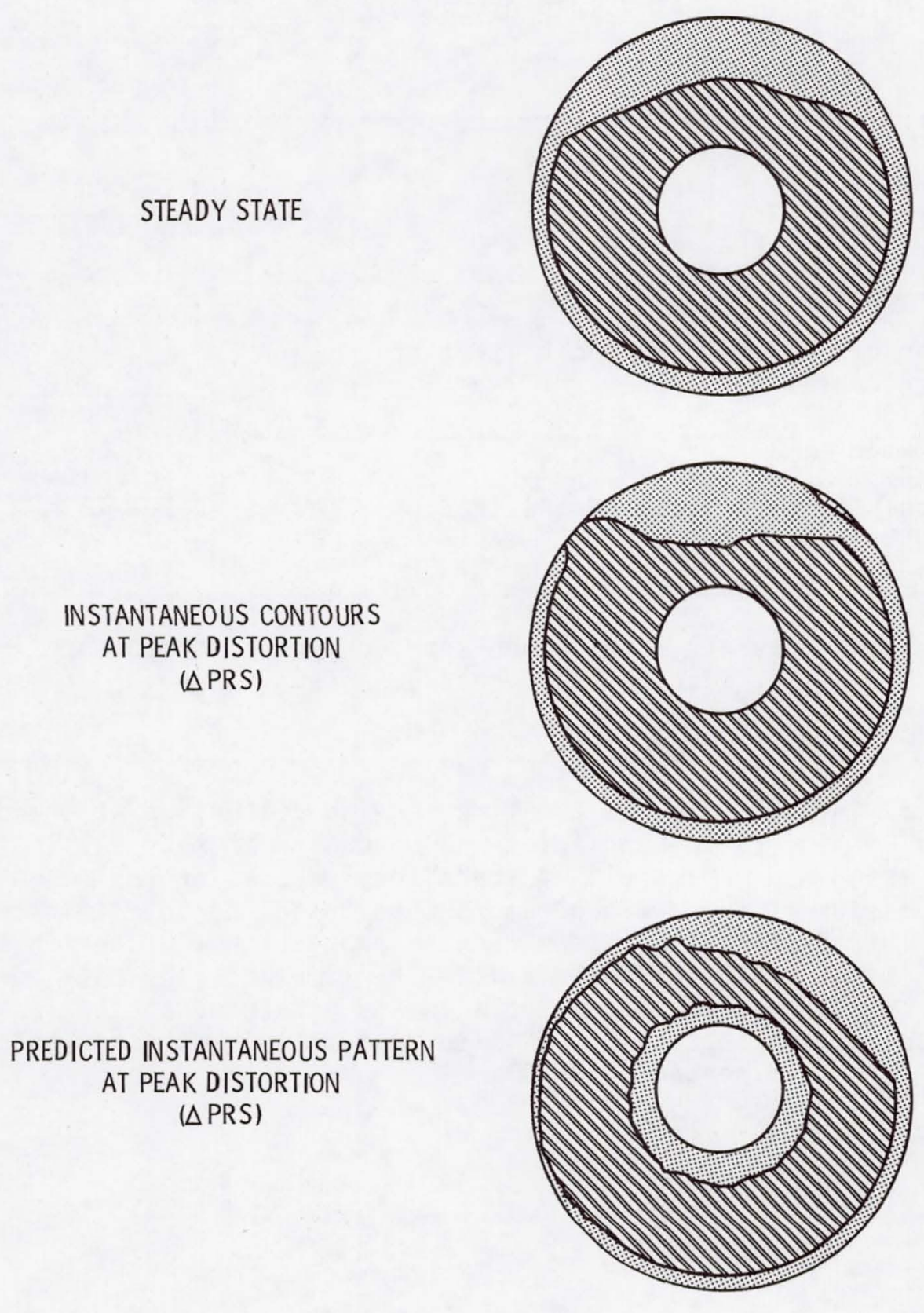

(d) Pitch $=0$ Yaw $=-5$.

Figure 13. - Concluded. 


\begin{tabular}{|c|c|c|c|}
\hline $\begin{array}{l}\text { 1. Report No. } \\
\text { NASA TM- } 83412\end{array}$ & 2. Government Accession No. & \multicolumn{2}{|c|}{ 3. Recipient's Catalog No. } \\
\hline \multicolumn{2}{|c|}{$\begin{array}{l}\text { 4. Title and Subtitle } \\
\text { DYNAMIC DISTORTION IN A SHORT S-SHAPED SUBSONIC } \\
\text { DIFFUSER WITH FLOW SEPARATION }\end{array}$} & \multicolumn{2}{|l|}{ 5. Report Date } \\
\hline \multicolumn{2}{|c|}{$\begin{array}{l}\text { 7. Author(s) } \\
\text { Richard Stumpf, Harvey E. Neumann, and } \\
\text { Charles C. Giamati }\end{array}$} & \multicolumn{2}{|c|}{$\begin{array}{l}\text { 8. Performing Organization Report No. } \\
\text { E-1695 }\end{array}$} \\
\hline \multicolumn{2}{|c|}{$\begin{array}{l}\text { 9. Performing Organization Name and Address } \\
\text { National Aeronautics and Space Administration } \\
\text { Lewis Research Center } \\
\text { Cleveland, Ohio } 44135\end{array}$} & \multicolumn{2}{|c|}{ 11. Contract or Grant No. } \\
\hline \multicolumn{2}{|c|}{$\begin{array}{l}\text { 12. Sponsoring Agency Name and Address } \\
\text { Nationa } 7 \text { Aeronautics and Space Administration } \\
\text { Washington, D.C. } 20546\end{array}$} & \multicolumn{2}{|c|}{ 14. Sponsoring Agency Code } \\
\hline \multicolumn{4}{|c|}{$\begin{array}{l}\text { 15. Supplementary Notes } \\
\text { Prepared for the Nineteenth Joint Propulsion Conference cosponsored by the AIAA, } \\
\text { SAE, and ASME, Seattle, Washington, June } 27-29,1983 \text {. }\end{array}$} \\
\hline \multicolumn{4}{|c|}{$\begin{array}{l}\text { 16. Abstract } \\
\text { An experimental investigation of the time varying distortion at the diffuser exit } \\
\text { of a subscale HiMAT forebody and inlet was conducted at Mach } 0.9 \text { in the Lewis } 8 \\
\text { by } 6 \text { Foot Supersonic Wind Tunnel. A transitory separation was detected within } \\
\text { the subsonic diffuser. Vortex generators were installed to eliminate the flow } \\
\text { separation. This paper will present results froma study of the instantaneous } \\
\text { pressure variations at the diffuser exit. The time unsteady total pressures at } \\
\text { the diffuser exit are computer interpolated and presented in the form of a movie } \\
\text { showing the transitory separation. Limited data showing the instantaneous } \\
\text { distortion levels is also presented. }\end{array}$} \\
\hline $\begin{array}{l}\text { 17. Key Words (Suggested by Author(s)) } \\
\text { Diffusers } \\
\text { Intakes } \\
\text { Supersonic inlets } \\
\text { Distortion }\end{array}$ & \begin{tabular}{r|r} 
18. Distrit \\
Unc \\
STA
\end{tabular} & $\begin{array}{l}\text { ed - unlimite } \\
\text { ory } 07\end{array}$ & \\
\hline $\begin{array}{l}\text { 19. Security Classif. (of this report) } \\
\text { Unclassified }\end{array}$ & $\begin{array}{l}\text { 0. Security Classif. (of this page) } \\
\text { Unclass ified }\end{array}$ & 21. No. of pages & 22. Price ${ }^{*}$ \\
\hline
\end{tabular}

*For sale by the National Technical Information Service, Springfield, Virginia 22161 